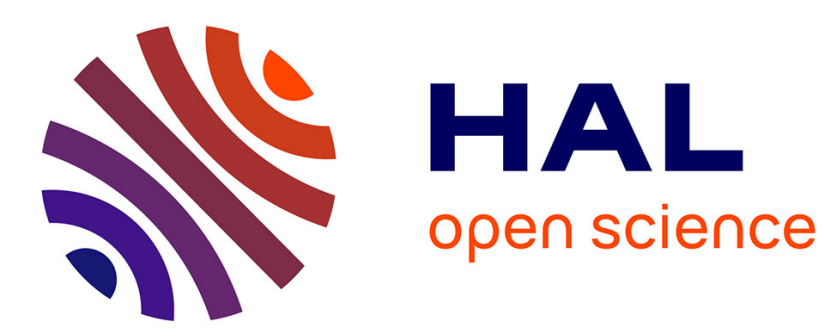

\title{
Comparison of NEWUOA with Different Numbers of Interpolation Points on the BBOB Noisy Testbed
}

Raymond Ros

\section{To cite this version:}

Raymond Ros. Comparison of NEWUOA with Different Numbers of Interpolation Points on the BBOB Noisy Testbed. Genetic and Evolutionary Computation Conference 2010, Jul 2010, Portland, OR, United States. inria-00473776

\section{HAL Id: inria-00473776 \\ https://hal.inria.fr/inria-00473776}

Submitted on 16 Apr 2010

HAL is a multi-disciplinary open access archive for the deposit and dissemination of scientific research documents, whether they are published or not. The documents may come from teaching and research institutions in France or abroad, or from public or private research centers.
L'archive ouverte pluridisciplinaire HAL, est destinée au dépôt et à la diffusion de documents scientifiques de niveau recherche, publiés ou non, émanant des établissements d'enseignement et de recherche français ou étrangers, des laboratoires publics ou privés. 


\title{
Comparison of NEWUOA with Different Numbers of Interpolation Points on the BBOB Noisy Testbed
}

\author{
Raymond Ros \\ TAO Team-Project - INRIA Saclay \\ LRI, Bat 490, Univ. Paris-Sud \\ F-91405 Orsay Cedex, France \\ raymond.ros@inria.fr
}

\begin{abstract}
In this paper, we study the performances of the NEW Unconstrained Optimization Algorithm (NEWUOA) with different numbers of interpolation points. NEWUOA is a trust region method, the number of points used to build the surrogate model is an input parameter of the algorithm. We compare the performances of NEWUOA using three different number of points in search spaces of dimension from two to forty on problems from the BBOB 2009 noisy function testbed. Using the maximum number of interpolation points grants the better results in this noisy setting.
\end{abstract}

\section{Categories and Subject Descriptors}

G.1.6 [Numerical Analysis]: Optimization-global optimization, unconstrained optimization; F.2.1 [Analysis of Algorithms and Problem Complexity]: Numerical Algorithms and Problems

\section{General Terms}

Algorithms

\section{Keywords}

Benchmarking, Black-box optimization

\section{INTRODUCTION}

The NEWUOA, for NEW Unconstrained Optimization Algorithm was introduced in [5] as a method for unconstrained derivative-free optimization. NEWUOA is a trustregion method which uses $m$ points to build a quadratic approximation of the objective function. The approximation is considered reliable within the radius of the current trust region. In this paper, we study the effect of the number of interpolation points $m$ on the performances of NEWUOA on a testbed of noisy functions.

We use three different values for $m$ which will be denoted NEWUOA, avg-NEWUOA and full-NEWUOA.

Permission to make digital or hard copies of all or part of this work for personal or classroom use is granted without fee provided that copies are not made or distributed for profit or commercial advantage and that copies bear this notice and the full citation on the first page. To copy otherwise, to republish, to post on servers or to redistribute to lists, requires prior specific permission and/or a fee.

GECCO'10, July 7-11, 2010, Portland, Oregon, USA.

Copyright 2010 ACM 978-1-4503-0073-5/10/07 ...\$10.00.
These variants are sorted by ascending numbers of interpolation points. The number of interpolation points of these variants depends on the dimension of the search space $n$. The variant denoted NEWUOA uses $2 n+1$ interpolation points as recommended in [5]. The avg-NEWUOA uses the rounded value of $\sqrt{(n+1 / 2)(n+1)(n+2)}$ interpolation points which is intermediate. The full-NEWUOA uses the maximum number $\frac{(n+1)(n+2)}{2}$. These three settings were already compared on a few test problems in [5].

The performances of the avg-NEWUOA are obtained on the BBOB 2009 testbed of noiseless functions. The avgNEWUOA is successively compared to NEWUOA and fullNEWUOA. The performances of both NEWUOA and fullNEWUOA on the BBOB 2009 noiseless functions were presented in [7].

\section{EXPERIMENTAL PROCEDURE}

To benchmark the avg-NEWUOA, we use the exact same experimental procedure that was presented in [7]. In particular the algorithm uses an independent multi-start procedure, as do NEWUOA and full-NEWUOA. The crafting effort [3] is equal to $\mathrm{CrE}=0$ for all three variants of the NEWUOA.

\section{RESULTS}

Results of the CPU-timing experiments are given in the paper benchmarking NEWUOA with all three settings of $m$ on the BBOB noiseless testbed submitted to the same workshop.

Results from experiments according to [3] on the benchmark functions given in $[1,4]$ are presented in this section. The Figures 1 and 2 and the Table 1 compare the avg-NEWUOA to NEWUOA. The Figures 3 and 4 and the Table 2 compare the avg-NEWUOA to full-NEWUOA. The expected running time (ERT), used in the figures and table, depends on a given target function value, $f_{\mathrm{t}}=f_{\mathrm{opt}}+$ $\Delta f_{\mathrm{t}}$, and is computed over all relevant trials as the number of function evaluations executed during each trial while the best function value did not reach $f_{\mathrm{t}}$, summed over all trials and divided by the number of trials that actually reached $f_{\mathrm{t}}$ $[3,6]$. Statistical significance is tested with the rank-sum test for a given target $\Delta f_{\mathrm{t}}\left(10^{-8}\right.$ in Figure 1) using, for each trial, either the number of needed function evaluations to reach $\Delta f_{\mathrm{t}}$ (inverted and multiplied by -1 ), or, if the target was not reached, the best $\Delta f$-value achieved, measured only up to the smallest number of overall function evaluations for any unsuccessful trial under consideration. 
The success probability of avg-NEWUOA reaching the precision $10^{-8}$ is only slightly larger than that of NEWUOA: in 5-D avg-NEWUOA is successful on 52 function instances (out of 450) in 5-D and NEWUOA only on 42; in 20-D avg-NEWUOA is successful on 30 function instances and NEWUOA only on 15 . On functions $f_{101}, f_{102}$ and $f_{103}$ which both NEWUOA and avg-NEWUOA solve in 5-D, the NEWUOA is slower faster than avg-NEWUOA.

To reach the precision $10^{-8}$, full-NEWUOA has the best success probability out of the three variants of NEWUOA: it solves 77 function instances in 5-D, 31 in 20-D. Also, the full-NEWUOA solves in 5-D the Rosenbrock function $f_{106}$ and the Sphere function $f_{109}$, both with moderate Cauchy noise, whereas avg-NEWUOA does not.

In 20-D, the NEWUOA only solves the Sphere function with moderate noise. The full-NEWUOA solves the Sphere function with all three noise models and is the fastest out of the three NEWUOA variants. In 20-D again, avg-NEWUOA does not solve $f_{103}$, NEWUOA does not solve neither $f_{102}$ nor $f_{103}$ and is the slowest out of the three variants of NEWUOA on $f_{101}$.

Overall the full-NEWUOA variant which uses the largest number of interpolation points performs better than the avgNEWUOA and the NEWUOA on the BBOB 2009 noisy testbed.

\section{REFERENCES}

[1] S. Finck, N. Hansen, R. Ros, and A. Auger. Real-parameter black-box optimization benchmarking 2010: Presentation of the noisy functions. Technical Report 2009/21, Research Center PPE, 2010.

[2] N. Hansen, A. Auger, S. Finck, and R. Ros. Real-parameter black-box optimization benchmarking 2009: Experimental setup. Technical Report RR-6828, INRIA, 2009.

[3] N. Hansen, A. Auger, S. Finck, and R. Ros. Real-parameter black-box optimization benchmarking 2010: Experimental setup. Technical Report RR-7215, INRIA, 2010.

[4] N. Hansen, S. Finck, R. Ros, and A. Auger. Real-parameter black-box optimization benchmarking 2009: Noisy functions definitions. Technical Report RR-6869, INRIA, 2009. Updated February 2010.

[5] M. J. D. Powell. The NEWUOA software for unconstrained optimization without derivatives. Large Scale Nonlinear Optimization, pages 255-297, 2006.

[6] K. Price. Differential evolution vs. the functions of the second ICEO. In Proceedings of the IEEE International Congress on Evolutionary Computation, pages 153-157, 1997.

[7] R. Ros. Benchmarking the NEWUOA on the BBOB-2009 noisy testbed. In F. Rothlauf, editor, GECCO (Companion), pages 2429-2434. ACM, 2009. 

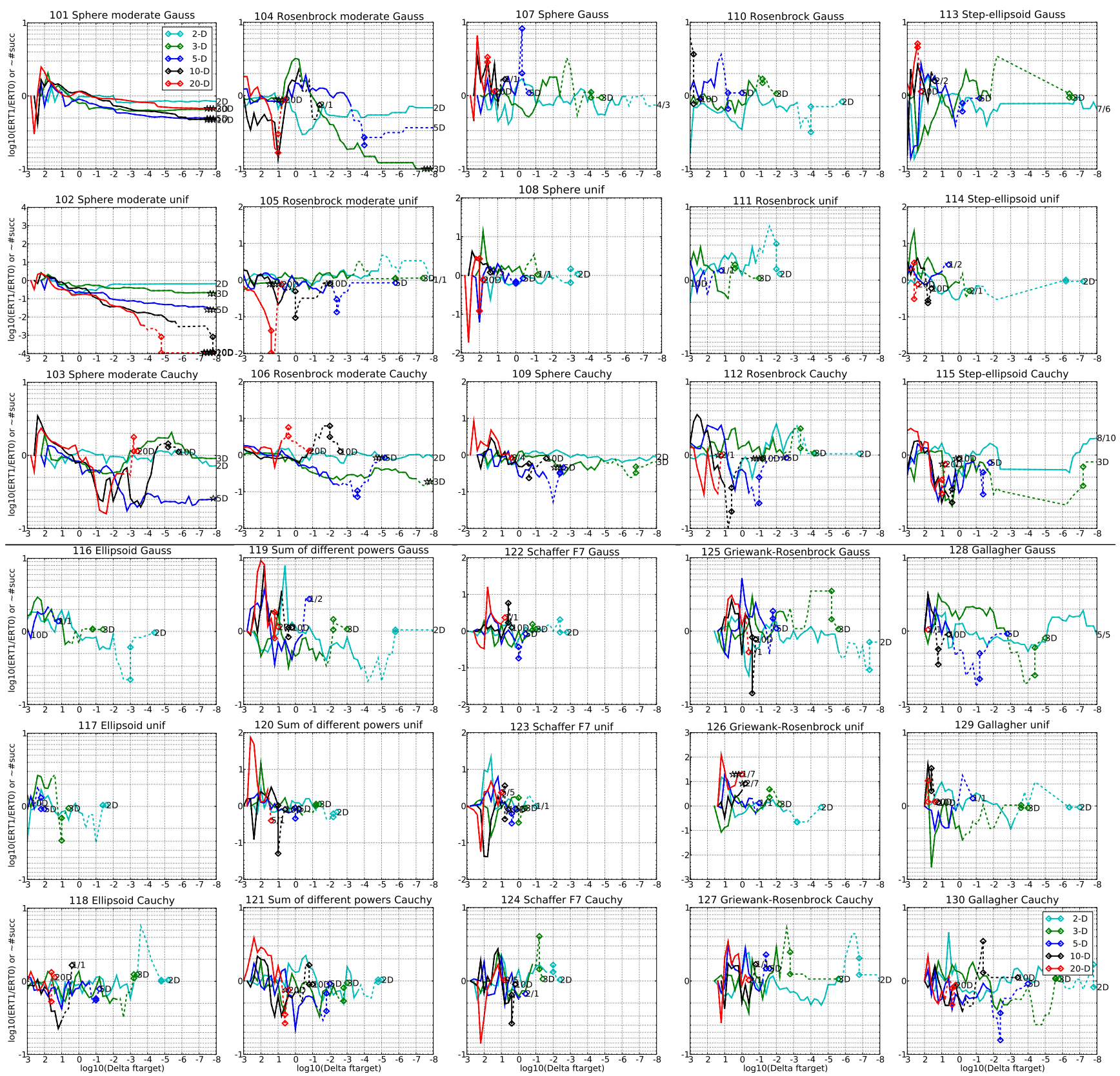

Figure 1: Ratio of the expected running times (ERT) of avg-NEWUOA divided by NEWUOA versus $\log _{10}(\Delta f)$ for $f_{101}-f_{130}$ in 2, 3, 5, 10, 20, 40-D. Ratios $<10^{\circ}$ indicate an advantage of avg-NEWUOA, smaller values are always better. The line gets dashed when for any algorithm the ERT exceeds thrice the median of the trial-wise overall number of $f$-evaluations for the same algorithm on this function. Symbols indicate the best achieved $\Delta f$-value of one algorithm (ERT gets undefined to the right). The dashed line continues as the fraction of successful trials of the other algorithm, where 0 means $0 \%$ and the y-axis limits mean $100 \%$, values below zero for avg-NEWUOA. The line ends when no algorithm reaches $\Delta f$ anymore. The number of successful trials is given, only if it was in $\{1 \ldots 9\}$ for avg-NEWUOA (1st number) and non-zero for NEWUOA (2nd number). Results are statistically significant with $p=0.05$ for one star and $p=10^{-\# \star}$ otherwise, with Bonferroni correction within each figure. 
$5-\mathrm{D}$
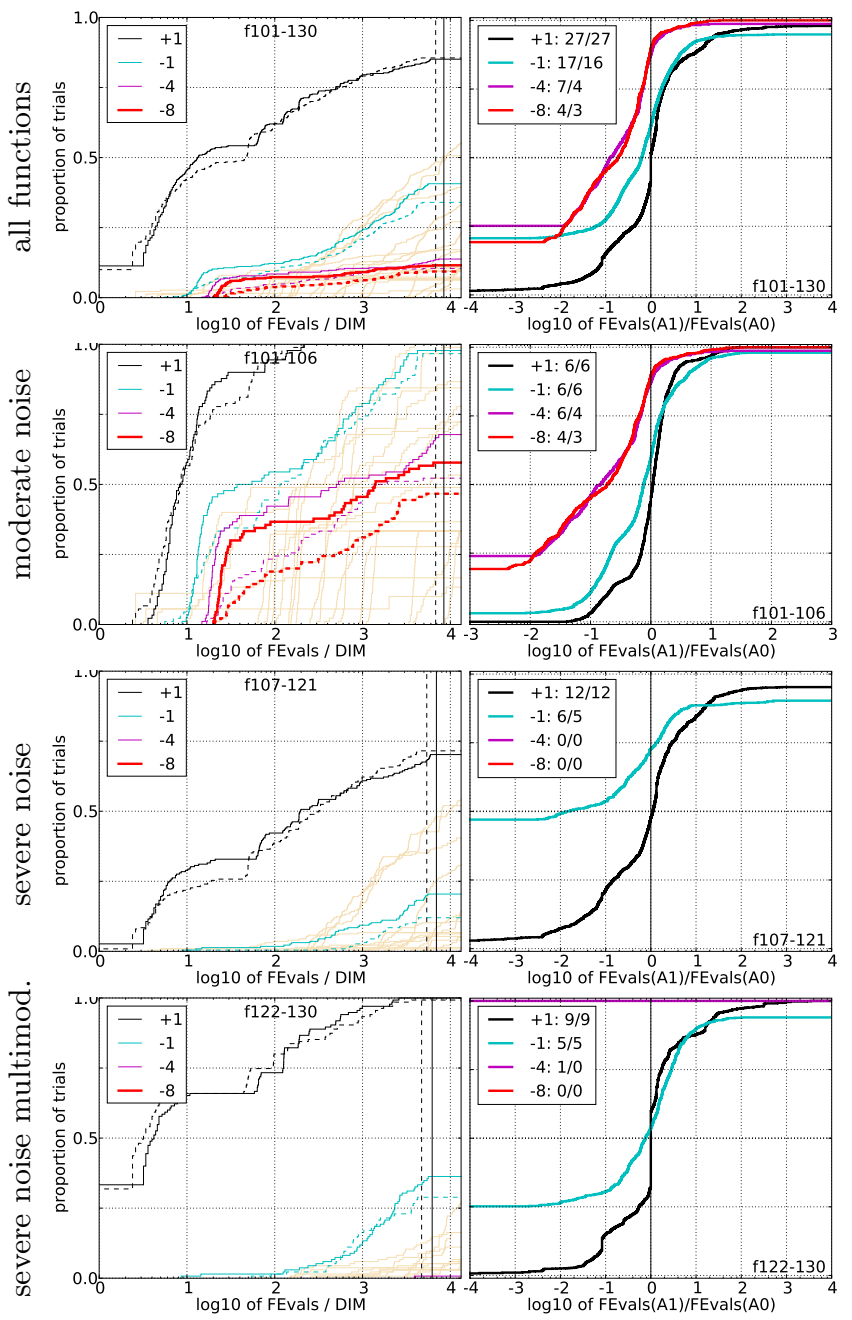

$20-\mathrm{D}$
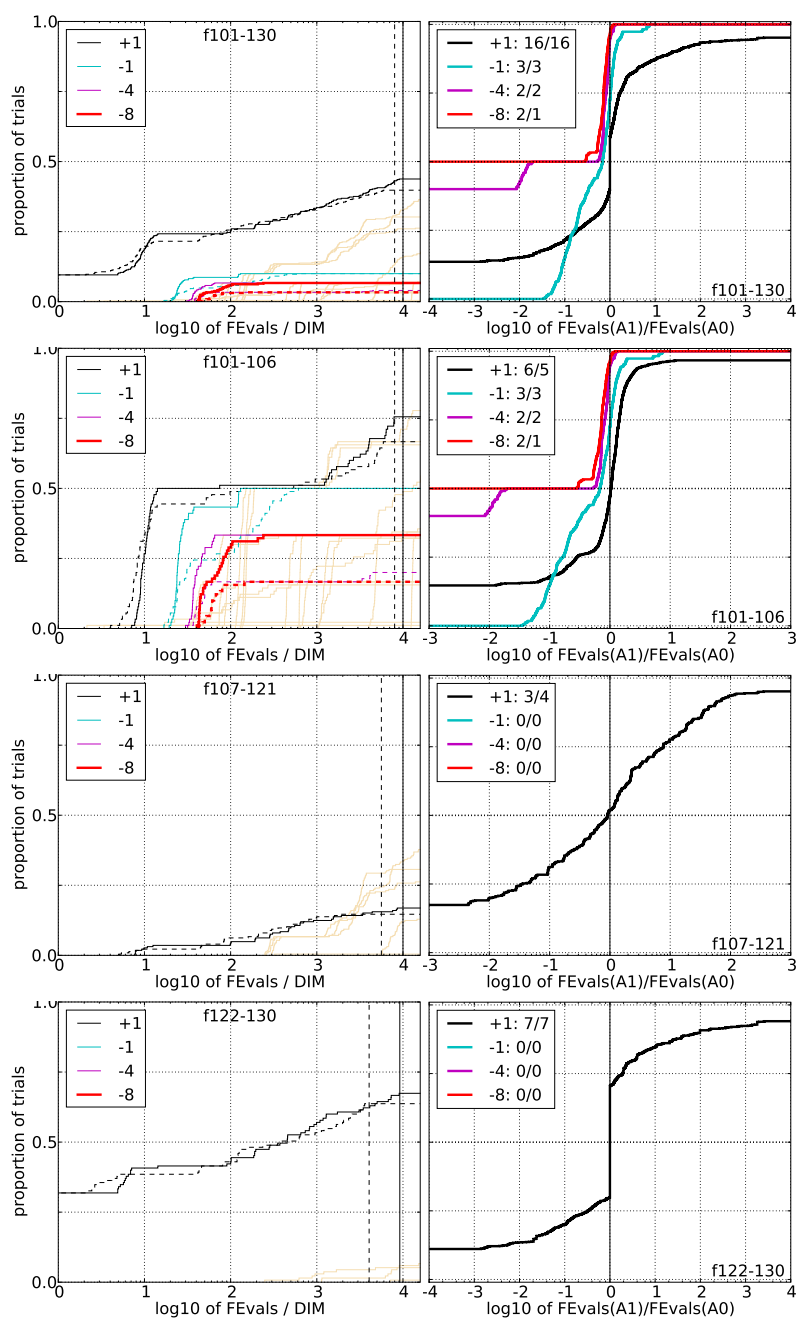

Figure 2: Empirical cumulative distributions (ECDF) of run lengths and speed-up ratios in 5-D (left) and 20-D (right). Left sub-columns: ECDF of the number of necessary function evaluations divided by dimension $D$ (FEvals/D) to reached a target value $f_{\text {opt }}+\Delta f$ with $\Delta f=10^{k}$, where $k \in\{1,-1,-4,-8\}$ is given by the first value in the legend, for avg-NEWUOA (solid) and NEWUOA (dashed). Light beige lines show the ECDF of FEvals for target value $\Delta f=10^{-8}$ of all algorithms benchmarked during BBOB-2009. Right sub-columns: ECDF of FEval ratios of avg-NEWUOA divided by NEWUOA, all trial pairs for each function. Pairs where both trials failed are disregarded, pairs where one trial failed are visible in the limits being $>0$ or $<1$. The legends indicate the number of functions that were solved in at least one trial (avg-NEWUOA first). 
$5-\mathrm{D}$

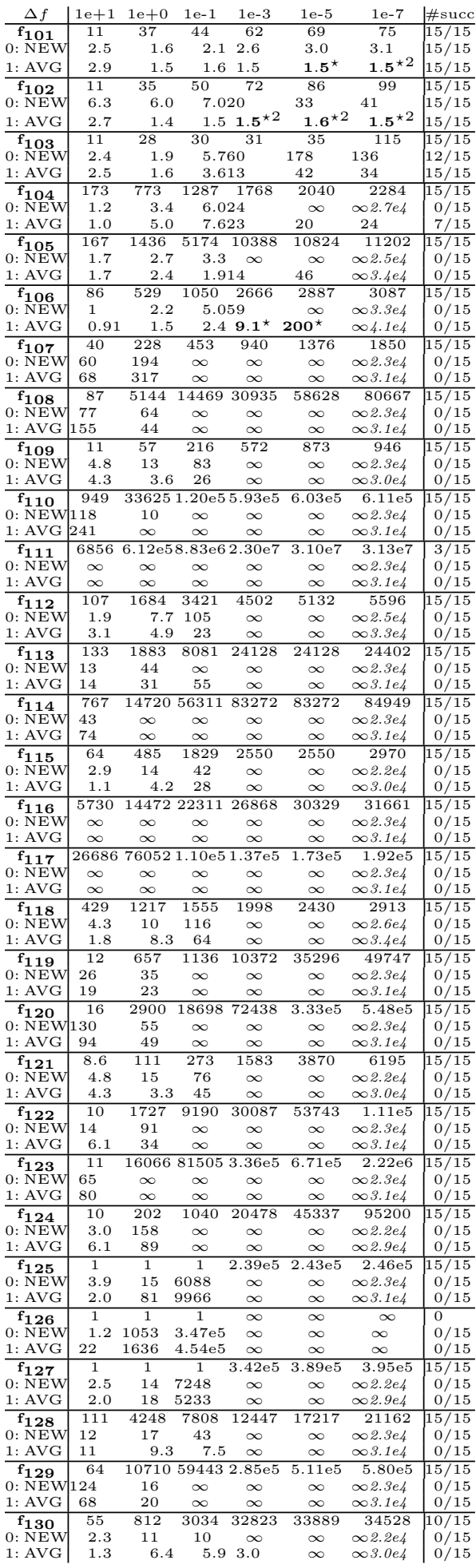

20-D

\begin{tabular}{|c|c|c|c|c|c|c|c|}
\hline$\Delta f$ & $e+1$ & $1 e+0$ & $1 \mathrm{e}-1$ & $1 e-3$ & $1 e-5$ & $1 e-7$ & \#succ \\
\hline 101 & 59 & 361 & 513 & 700 & 739 & 783 & $15 / 15$ \\
\hline $0: \mathrm{NEW}$ & 3.1 & 1 & 1 & 1.1 & 1.5 & 1.6 & $15 / 15$ \\
\hline $1: \mathrm{AVG}$ & 3.3 & 1.2 & 0.95 & 0.94 & $1.0^{\star 2}$ & $1.1^{\star 2}$ & $15 / 15$ \\
\hline f 100 & 231 & 399 & 579 & 921 & 1157 & 1407 & $15 / 15$ \\
\hline $0: \mathrm{NEW}$ & 2.9 & 6.1 & 6.3 & 45 & $\infty$ & $\infty 1.0 e 5$ & $0 / 15$ \\
\hline 1: AVG & 0.92 & 1.1 & $0.93^{\star}$ & $0.88^{\star}$ & $1.1^{\star 3}$ & $1.3^{\star 3}$ & $15 / 15$ \\
\hline$f_{103}$ & 65 & 417 & 629 & 1313 & 1893 & 2464 & $14 / 15$ \\
\hline $0: \mathrm{NEW}$ & 2.3 & 1 & 5.9 & 1231 & $\infty$ & $\infty 1.1 \mathrm{e} 5$ & $0 / 15$ \\
\hline $1: \mathrm{AVG}$ & 3.0 & 0.95 & 2.0 & 655 & $\infty$ & $\infty 2.0 e 5$ & $0 / 15$ \\
\hline$f_{104}$ & 23690 & 85656 & $1.71 \mathrm{e} 5$ & $1.82 \mathrm{e} 5$ & $1.89 \mathrm{e} 5$ & $1.96 \mathrm{e} 5$ & $15 / 15$ \\
\hline $0: \mathrm{NEW}$ & 68 & $\infty$ & $\infty$ & $\infty$ & $\infty$ & $\infty 1.1 e 5$ & $0 / 15$ \\
\hline $1: \mathrm{AVG}$ & 11 & $\infty$ & $\infty$ & $\infty$ & $\infty$ & $\infty 2.0 e 5$ & $0 / 15$ \\
\hline$f_{105}$ & $1.92 \mathrm{e} 5$ & $6.11 \mathrm{e} 5$ & $6.32 \mathrm{e} 5$ & $6.49 \mathrm{e} 5$ & $6.60 \mathrm{e} 5$ & $6.70 \mathrm{e} 5$ & $15 / 15$ \\
\hline $0: \mathrm{NEW}$ & $\infty$ & $\infty$ & $\infty$ & $\infty$ & $\infty$ & $\infty 9.4 e 4$ & $0 / 15$ \\
\hline 1: AVG & $7.2^{\star 3}$ & $\infty$ & $\infty$ & $\infty$ & $\infty$ & $\infty 2.0 e 5$ & $0 / 15$ \\
\hline & 11480 & 21668 & 23746 & 25470 & 26492 & 27360 & $15 / 15$ \\
\hline $0: N E W$ & 7.0 & 31 & $\infty$ & $\infty$ & $\infty$ & $\infty 1.5 \mathrm{e} 5$ & $0 / 15$ \\
\hline 1: AVG & 8.1 & $\infty$ & $\infty$ & $\infty$ & $\infty$ & $\infty 2.0 e 5$ & $0 / 15$ \\
\hline & 8571 & 13582 & 16226 & 27357 & 52486 & 65052 & $15 / 15$ \\
\hline NEW & $\infty$ & $\infty$ & $\infty$ & $\infty$ & $\infty$ & $\infty 7.8 e 4$ & $0 / 15$ \\
\hline 1: AVG & $\infty$ & $\infty$ & $\infty$ & $\infty$ & $\infty$ & $\infty 1.8 \mathrm{e} 5$ & $0 / 15$ \\
\hline$f_{108}$ & 58063 & 97228 & $2.03 \mathrm{e} 5$ & $4.46 \mathrm{e} 5$ & $6.30 \mathrm{e} 5$ & $8.98 \mathrm{e} 5$ & $15 / 15$ \\
\hline $0: \mathrm{NEW}$ & $\infty$ & $\infty$ & $\infty$ & $\infty$ & $\infty$ & $\infty 7.8 e 4$ & $0 / 15$ \\
\hline 1: AVG & $\infty$ & $\infty$ & $\infty$ & $\infty$ & $\infty$ & $\infty 1.8 \mathrm{e} 5$ & $0 / 15$ \\
\hline$f_{109}$ & 333 & 632 & 1138 & 2287 & 3583 & 4952 & $15 / 15$ \\
\hline $0: \mathrm{NEW}$ & 17 & $\infty$ & $\infty$ & $\infty$ & $\infty$ & $\infty 8.4 e 4$ & $0 / 15$ \\
\hline 1: AVG & 17 & $\infty$ & $\infty$ & $\infty$ & $\infty$ & $\infty 1.9 \mathrm{e} 5$ & $0 / 15$ \\
\hline$f_{110}$ & $\infty$ & $\infty$ & $\infty$ & $\infty$ & $\infty$ & $\infty$ & \\
\hline $0: \mathrm{NEW}$ & $\infty$ & $\infty$ & $\infty$ & $\infty$ & $\infty$ & $\infty$ & $0 / 15$ \\
\hline $1: \mathrm{AVG}$ & $\infty$ & $\infty$ & $\infty$ & $\infty$ & $\infty$ & $\infty$ & $0 / 15$ \\
\hline$f_{111}$ & $\infty$ & $\infty$ & $\infty$ & $\infty$ & $\infty$ & $\infty$ & 0 \\
\hline $0: \mathrm{NEW}$ & $\infty$ & $\infty$ & $\infty$ & $\infty$ & $\infty$ & $\infty$ & $0 / 15$ \\
\hline 1: AVG & $\infty$ & $\infty$ & $\infty$ & $\infty$ & $\infty$ & $\infty$ & $0 / 15$ \\
\hline$f_{112}$ & 25552 & 64124 & 69621 & 73557 & 76137 & 78238 & $15 / 15$ \\
\hline $0: \mathrm{NEW}$ & $\infty$ & $\infty$ & $\infty$ & $\infty$ & $\infty$ & $\infty 9.8 e 4$ & $0 / 15$ \\
\hline 1: $\mathrm{AVG}$ & $\infty$ & $\infty$ & $\infty$ & $\infty$ & $\infty$ & $\infty 2.0 e 5$ & $0 / 15$ \\
\hline & 50123 & $3.64 \mathrm{e} 5$ & $5.60 \mathrm{e} 5$ & $5.88 \mathrm{e} 5$ & $5.88 \mathrm{e} 5$ & $5.91 \mathrm{e} 5$ & $15 / 15$ \\
\hline $0: \mathrm{NEW}$ & $\infty$ & $\infty$ & $\infty$ & $\infty$ & $\infty$ & $\infty 7.8 e 4$ & $0 / 15$ \\
\hline 1: AVG & $\infty$ & $\infty$ & $\infty$ & $\infty$ & $\infty$ & $\infty 1.8 \mathrm{e} 5$ & $0 / 15$ \\
\hline$f_{114}$ & $2.08 \mathrm{e} 5$ & $1.12 \mathrm{e} 6$ & $1.45 \mathrm{e} 6$ & $1.57 \mathrm{e} 6$ & $1.57 \mathrm{e} 6$ & $1.58 \mathrm{e} 6$ & $15 / 15$ \\
\hline $\mathrm{NEW}$ & $\infty$ & $\infty$ & $\infty$ & $\infty$ & $\infty$ & $\infty 7.8 e 4$ & $0 / 15$ \\
\hline 1: AVG & $\infty$ & $\infty$ & $\infty$ & $\infty$ & $\infty$ & $\infty 1.8 \mathrm{e} 5$ & $0 / 15$ \\
\hline & 2405 & 30268 & 91749 & $1.27 \mathrm{e} 5$ & $1.27 \mathrm{e} 5$ & $1.29 \mathrm{e} 5$ & $15 / 15$ \\
\hline IEW & 236 & $\infty$ & $\infty$ & $\infty$ & $\infty$ & $\infty 8.5 e 4$ & $0 / 15$ \\
\hline $1: \mathrm{AVG}$ & 108 & $\infty$ & $\infty$ & $\infty$ & $\infty$ & $\infty 1.9 \mathrm{e} 5$ & $0 / 15$ \\
\hline & $4.98 \mathrm{e} 5$ & $6.94 \mathrm{e} 5$ & $8.93 \mathrm{e} 5$ & $1.03 \mathrm{e} 6$ & $1.08 \mathrm{e} 6$ & $1.12 \mathrm{e} 6$ & $15 / 15$ \\
\hline $\mathrm{NEW}$ & $\infty$ & $\infty$ & $\infty$ & $\infty$ & $\infty$ & $\infty 7.7 e 4$ & $0 / 15$ \\
\hline $1: \mathrm{AVG}$ & $\infty$ & $\infty$ & $\infty$ & $\infty$ & $\infty$ & $\infty 1.8 \mathrm{e} 5$ & $0 / 15$ \\
\hline & $1.79 \mathrm{e} 6$ & $2.46 \mathrm{e} 6$ & $2.60 \mathrm{e} 6$ & $2.91 \mathrm{e} 6$ & $3.24 \mathrm{e} 6$ & $3.62 \mathrm{e} 6$ & $15 / 15$ \\
\hline 0 : NEW & $\infty$ & $\infty$ & $\infty$ & $\infty$ & $\infty$ & $\infty 7.7 e 4$ & $0 / 15$ \\
\hline $1: \mathrm{AVG}$ & $\infty$ & $\infty$ & $\infty$ & $\infty$ & $\infty$ & $\infty 1.8 \mathrm{e} 5$ & $0 / 15$ \\
\hline & 6908 & 11786 & 17514 & 26342 & 30062 & 32659 & $15 / 15$ \\
\hline $0: \mathrm{NEW}$ & $\infty$ & $\infty$ & $\infty$ & $\infty$ & $\infty$ & $\infty 1.1 e 5$ & $0 / 15$ \\
\hline 1: AVG & $\infty$ & $\infty$ & $\infty$ & $\infty$ & $\infty$ & $\infty 2.0 e 5$ & $0 / 15$ \\
\hline & 2771 & 29365 & 35930 & $4.11 \mathrm{e} 5$ & $1.40 \mathrm{e} 6$ & $1.90 \mathrm{e} 6$ & $15 / 15$ \\
\hline $\mathrm{NEW}$ & 398 & $\infty$ & $\infty$ & $\infty$ & $\infty$ & $\infty 7.8 e 4$ & $0 / 15$ \\
\hline 1: AVG & $\infty$ & $\infty$ & $\infty$ & $\infty$ & $\infty$ & $\infty 1.8 \mathrm{e} 5$ & $0 / 15$ \\
\hline$f_{120}$ & 36040 & $1.79 \mathrm{e} 5$ & $2.81 \mathrm{e} 5$ & $1.59 \mathrm{e} 6$ & $6.74 \mathrm{e} 6$ & $1.35 \mathrm{e} 7$ & $13 / 15$ \\
\hline & $\infty$ & $\infty$ & $\infty$ & $\infty$ & $\infty$ & $\infty 7.8 e 4$ & $0 / 15$ \\
\hline 1: AVG & $\infty$ & $\infty$ & $\infty$ & $\infty$ & $\infty$ & $\infty 1.8 \mathrm{e} 5$ & 15 \\
\hline$f_{121}$ & 249 & 769 & 1426 & 9304 & 34434 & 57404 & $15 / 15$ \\
\hline $\mathrm{w}$ & 31 & $\infty$ & $\infty$ & $\infty$ & $\infty$ & $\infty 8$. & $0 / 15$ \\
\hline 1: AVG & 49 & $\infty$ & $\infty$ & $\infty$ & $\infty$ & $\infty 1.8 \mathrm{e} 5$ & $0 / 15$ \\
\hline$f_{122}$ & 692 & 52008 & $1.40 \mathrm{e} 5$ & $7.93 \mathrm{e} 5$ & $2.00 \mathrm{e} 6$ & $5.82 \mathrm{e} 6$ & $15 / 15$ \\
\hline & 82 & $\infty$ & $\infty$ & $\infty$ & $\infty$ & $\infty 7$ & $0 / 15$ \\
\hline $1: \mathrm{AVG}$ & 125 & $\infty$ & $\infty$ & $\infty$ & $\infty$ & $\infty 1.8 e^{5}$ & $0 / 15$ \\
\hline$f_{123}$ & 1063 & $5.30 \mathrm{e} 5$ & $1.49 \mathrm{e} 6$ & $5.29 \mathrm{e} 6$ & $2.71 \mathrm{e} 7$ & $1.58 \mathrm{e} 8$ & 0 \\
\hline & 174 & $\infty$ & $\infty$ & $\infty$ & $\infty$ & $\infty 7.8 e 4$ & $0 / 15$ \\
\hline 1: AVG & 405 & $\infty$ & $\alpha$ & $\infty$ & $\infty$ & $\infty 1$. & 1 \\
\hline & 192 & 1959 & 40840 & $1.27 \mathrm{e}^{5}$ & $3.89 \mathrm{e} 5$ & $7.99 \mathrm{e} 5$ & 15 \\
\hline $\mathrm{EW}$ & 91 & $\infty$ & $\infty$ & $\infty$ & $\infty$ & $\infty 7.8 e 4$ & $0 / 15$ \\
\hline $1: \mathrm{AVG}$ & 95 & $\infty$ & $\infty$ & $\infty$ & $\infty$ & $\infty 1.8 e^{2}$ & 0 \\
\hline$f_{125}$ & 1 & 1 & 4 & $2.50 \mathrm{e} 7$ & $8.03 \mathrm{e} 7$ & $8.06 \mathrm{e} 7$ & $4 / 15$ \\
\hline & 1 & & $\infty$ & $\infty$ & $\infty$ & $\infty 7.8 e 4$ & $0 / 15$ \\
\hline 1: AVG & 1 & & $\infty$ & $\infty$ & $\infty$ & $\infty 1.8 \mathrm{e} 5$ & $0 / 15$ \\
\hline$f_{126}$ & 1 & 1 & 1 & $\infty$ & $\infty$ & $\infty$ & 0 \\
\hline NEW & 4.2 & $1.32 \mathrm{e} 5^{\star}$ & $\infty$ & $\infty$ & & $\infty$ & \\
\hline $1: \mathrm{AVG}$ & 122 & $2.60 \mathrm{e} 6$ & $\infty$ & $\infty$ & $\infty$ & $\infty$ & $0 / 15$ \\
\hline & 1 & 1 & 1 & $4.43 \mathrm{e} 6$ & $7.27 \mathrm{e} 6$ & $7.43 \mathrm{e} 6$ & $15 / 15$ \\
\hline & 3.7 & & & $\infty$ & $\propto$ & $\infty 7$ & \\
\hline 1: AVG & 7.7 & 219 & $\infty$ & $\infty$ & $\infty$ & $\infty 1.8 \mathrm{e} 5$ & $0 / 15$ \\
\hline$f_{128}$ & $1.40 \mathrm{e} 5$ & $1.34 \mathrm{e} 7$ & $1.72 \mathrm{e} 7$ & $1.72 \mathrm{e} 7$ & $1.72 \mathrm{e} 7$ & $1.72 \mathrm{e} 7$ & $9 / 15$ \\
\hline & . & $\infty$ & $\infty$ & $\infty$ & $\alpha$ & $\infty 7$ & $0 /$ \\
\hline 1: AVG & $\infty$ & $\infty$ & $\infty$ & $\infty$ & $\infty$ & $\infty 1.8 \mathrm{e} 5$ & $0 / 15$ \\
\hline & $7.81 \mathrm{e}$ & $4.13 \mathrm{e} 7$ & $4.15 \mathrm{e} 7$ & $4.18 \mathrm{e} 7$ & $4.21 \mathrm{e} 7$ & $4.24 \mathrm{e} 7$ & $5 / 15$ \\
\hline $\mathrm{NEW}$ & 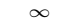 & $\infty$ & $\infty$ & $\infty$ & $\infty$ & $\infty 7.8 e 4$ & $0 / 15$ \\
\hline $1: \mathrm{AVG}$ & $\infty$ & $\infty$ & $\infty$ & $\infty$ & $\infty$ & $\infty 1.8 \mathrm{e} 5$ & $0 / 15$ \\
\hline & 4904 & 93149 & $2.52 \mathrm{e} 5$ & $2.54 \mathrm{e} 5$ & $2.55 \mathrm{e} 5$ & $2.57 \mathrm{e} 5$ & $7 / 15$ \\
\hline & 9.1 & 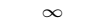 & $\infty$ & $\infty$ & $\infty$ & $\infty 8.0 e 4$ & $0 / 15$ \\
\hline 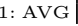 & 6.6 & $\infty$ & $\infty$ & $\infty$ & $\infty$ & $\infty 1.8 \mathrm{e} 5$ & $0 / 15$ \\
\hline
\end{tabular}

Table 1: ERT in number of function evaluations divided by the best ERT measured during BBOB-2009 (given in the respective first row) for different $\Delta f$ values for functions $f_{101}-f_{130}$. \#succ is the number of trials that reached the final target $f_{\mathrm{opt}}+10^{-8}$. 0: NEW is NEWUOA and 1: AVG is avg-NEWUOA. \#succ is the number of trials that reached the final target $f_{\mathrm{opt}}+10^{-8}$. 0: NEW is NEWUOA and 1: AVG is avg-NEWUOA. Bold entries are statistically significantly better compared to the other algorithm, with $p=0.05$ or $p=10^{-k}$ where $k>1$ is the number following the $\star$ symbol, with Bonferroni correction of 60 . 

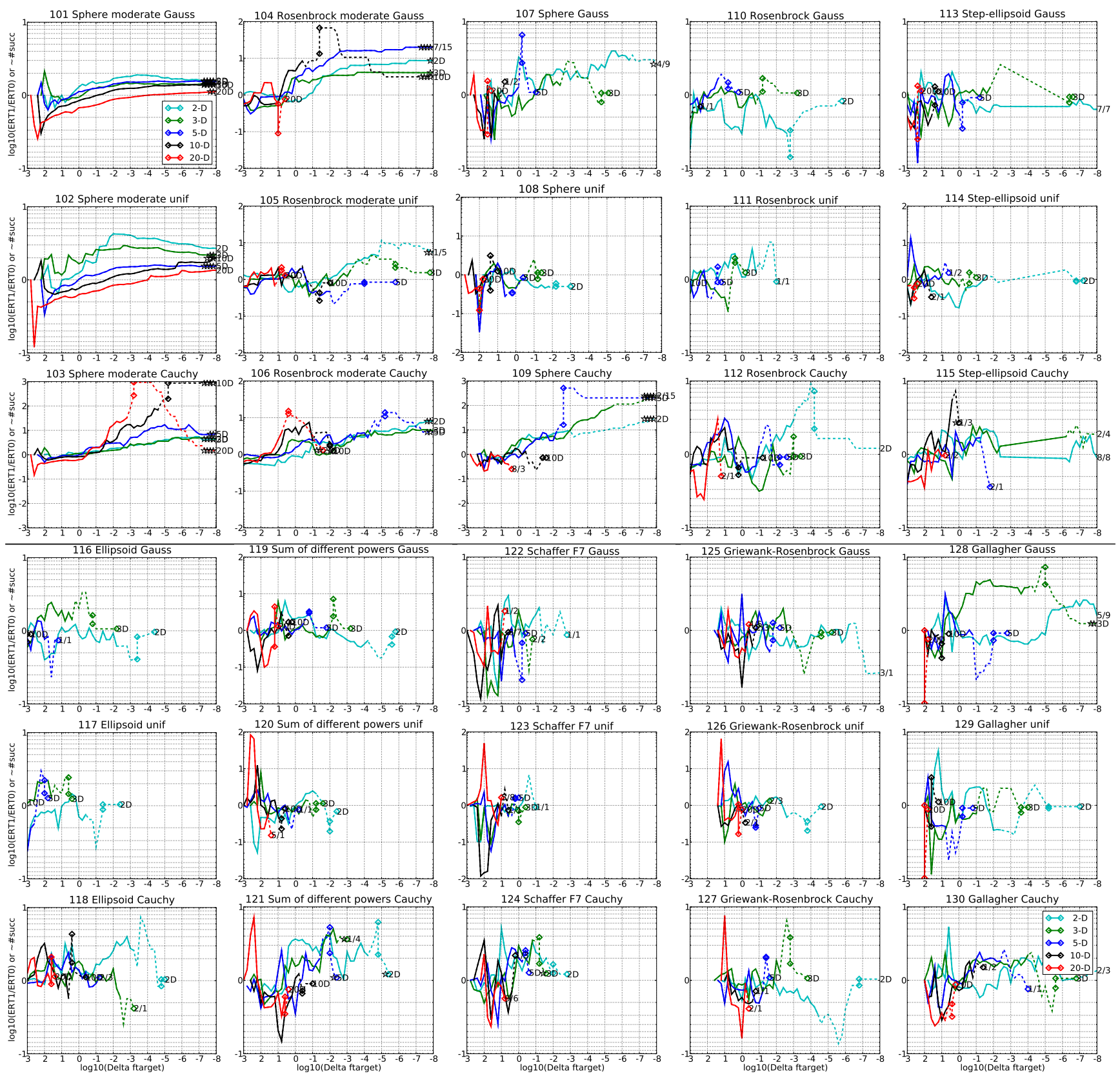

Figure 3: Ratio of the expected running times (ERT) of avg-NEWUOA divided by full-NEWUOA versus $\log _{10}(\Delta f)$ for $f_{101}-f_{130}$ in $2,3,5,10,20,40$-D. Ratios $<10^{0}$ indicate an advantage of avg-NEWUOA, smaller values are always better. The line gets dashed when for any algorithm the ERT exceeds thrice the median of the trial-wise overall number of $f$-evaluations for the same algorithm on this function. Symbols indicate the best achieved $\Delta f$-value of one algorithm (ERT gets undefined to the right). The dashed line continues as the fraction of successful trials of the other algorithm, where 0 means $0 \%$ and the $y$-axis limits mean $100 \%$, values below zero for avg-NEWUOA. The line ends when no algorithm reaches $\Delta f$ anymore. The number of successful trials is given, only if it was in $\{1 \ldots 9\}$ for avg-NEWUOA (1st number) and non-zero for full-NEWUOA (2nd number). Results are statistically significant with $p=0.05$ for one star and $p=10^{-\# \star}$ otherwise, with Bonferroni correction within each figure. 
$5-\mathrm{D}$
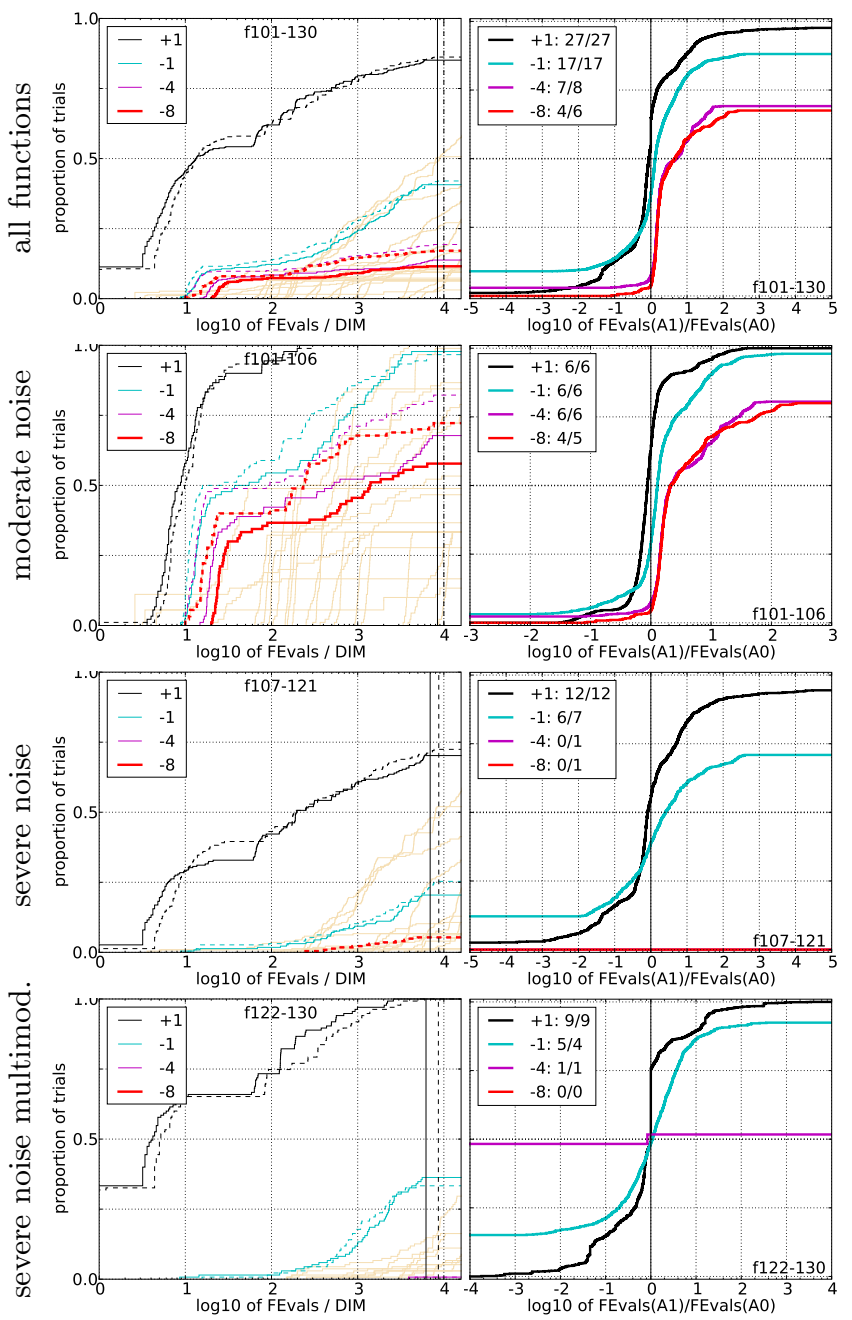

$20-\mathrm{D}$
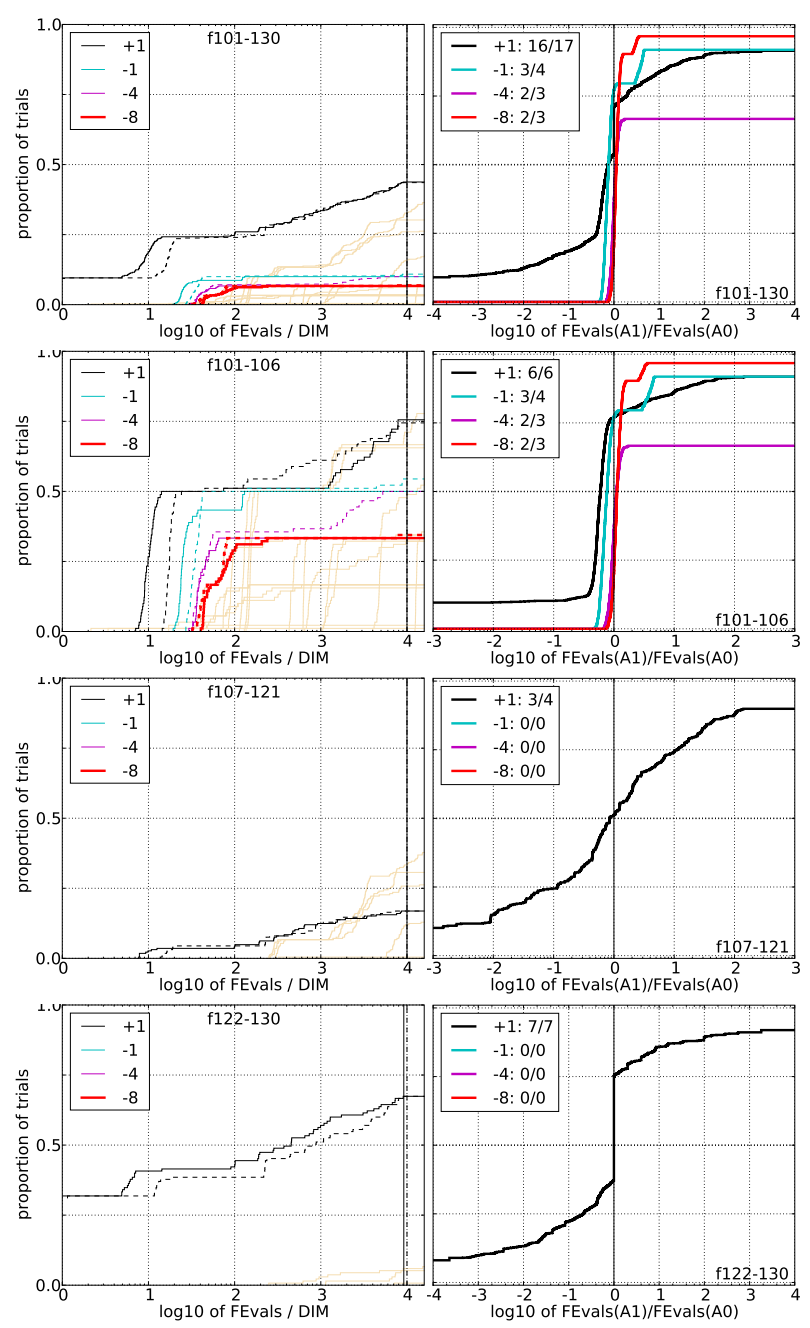

Figure 4: Empirical cumulative distributions (ECDF) of run lengths and speed-up ratios in 5-D (left) and 20-D (right). Left sub-columns: ECDF of the number of necessary function evaluations divided by dimension $D$ (FEvals/D) to reached a target value $f_{\text {opt }}+\Delta f$ with $\Delta f=10^{k}$, where $k \in\{1,-1,-4,-8\}$ is given by the first value in the legend, for avg-NEWUOA (solid) and full-NEWUOA (dashed). Light beige lines show the ECDF of FEvals for target value $\Delta f=10^{-8}$ of all algorithms benchmarked during BBOB-2009. Right sub-columns: ECDF of FEval ratios of avg-NEWUOA divided by full-NEWUOA, all trial pairs for each function. Pairs where both trials failed are disregarded, pairs where one trial failed are visible in the limits being $>0$ or $<1$. The legends indicate the number of functions that were solved in at least one trial (avg-NEWUOA first). 
$5-\mathrm{D}$

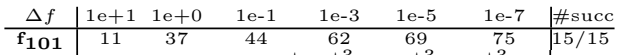

\begin{tabular}{c|cccccc|c}
\hline $\mathbf{f}_{\mathbf{1 0 1}}$ & 11 & 37 & 44 & 62 & 69 & $\mathbf{7 5}$ & $15 / 15$ \\
0: FUL & 3.3 & 1.3 & $\mathbf{1 . 3}^{\star}$ & $\mathbf{1}^{\star 3}$ & $\mathbf{1}^{\star 3}$ & $\mathbf{1}^{\star 3}$ & $15 / 15$ \\
1: AVG & 2.9 & 1.5 & 1.6 & 1.5 & 1.5 & 1.5 & $15 / 15$ \\
\hline f 102 & 11 & 35 & 50 & 72 & 86 & 99 & $15 / 15$ \\
0: FUL & 3.2 & 1.4 & 1.2 & $\mathbf{1}^{\star 2}$ & $\mathbf{1}^{\star 2}$ & $\mathbf{1}^{\star 2}$ & $15 / 15$ \\
1: AVG & 2.7 & 1.4 & 1.5 & 1.5 & 1.6 & 1.5 & $15 / 15$ \\
\hline
\end{tabular}

\begin{tabular}{c|cccccc|c}
$1: \mathrm{AVG}$ & 2.7 & 1.4 & 1.5 & 1.5 & 1.6 & 1.5 & $15 / 15$ \\
\hline $\mathbf{f}_{103}$ & 11 & 28 & 30 & 31 & 35 & 115 & $15 / 15$
\end{tabular}

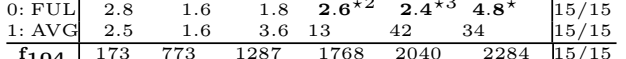

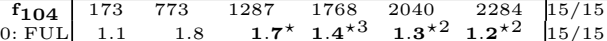

\begin{tabular}{c|ccccccc|r} 
1: AVG & 1.0 & 5.0 & 7.6 & 23 & 20 & 24 & $7 / 15$ \\
\hline$f_{105}$ & 167 & 1436 & 5174 & 10388 & 10824 & 11202 & $15 / 15$
\end{tabular}

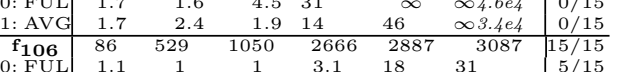

\begin{tabular}{l|llcccc|c} 
0: FUL & 1.1 & 1 & 1 & 3.1 & 18 & 31 & $5 / 15$ \\
1: AVG & 0.91 & 1.5 & 2.4 & 9.1 & 200 & $\infty 4.1 e 4$ & $0 / 15$ \\
\hline$f_{107}$ & 40 & 228 & 453 & 940 & 1376 & 1850 & $15 / 15$
\end{tabular}

\begin{tabular}{c|cccccc|r}
\hline$f_{107}$ & 40 & 228 & 453 & 940 & 1376 & 1850 & $15 / 15$ \\
0: FUL & 85 & 247 & 1373 & $\infty$ & $\infty$ & $\infty 4.2 e 4$ & $0 / 15$
\end{tabular}

\begin{tabular}{c|cccccc|c} 
1: AVG & 68 & 317 & $\infty$ & $\infty$ & $\infty$ & $\infty 3.1 e 4$ & $0 / 15$ \\
\hline$f_{108}$ & 87 & 5144 & 14469 & 30935 & 58628 & 80667 & $15 / 15$
\end{tabular}

\begin{tabular}{c|cccccc|c}
$0:$ FUL & 78 & $\infty$ & $\infty$ & $\infty$ & $\infty$ & $\infty 4.3 e 4$ & $0 / 15$ \\
1: AVG & 155 & 44 & $\infty$ & $\infty$ & $\infty$ & $\infty 3.1 e 4$ & $0 / 15$ \\
\hline$f_{109}$ & 11 & 57 & 216 & 572 & 873 & 946 & $15 / 15$
\end{tabular}

\begin{tabular}{c|cccccc|c}
\hline f $_{109}$ & 11 & 57 & 216 & 572 & 873 & 946 & $15 / 15$ \\
0: FUL & 5.6 & 5.0 & 6.1 & $\mathbf{2 1}^{\star 3}$ & $\mathbf{2 2}^{\star 3}$ & $\mathbf{2 0}^{\star 3}$ & $12 / 15$ \\
\hline . & .3 & 3.6 & 26 & & &
\end{tabular}

\begin{tabular}{l|rrrrrrrr}
1 : AVG & 4.3 & 3.6 & 26 & $\infty$ & $\infty$ & $\infty 3.0 \mathrm{e} 4$ & $0 / 15$ \\
\hline $\mathrm{f}_{110}$ & 949 & 33625 & $1.20 \mathrm{e} 5$ & $5.93 \mathrm{e} 5$ & $6.03 \mathrm{e} 5$ & $6.11 \mathrm{e} 5$ & $15 / 15$
\end{tabular}

\begin{tabular}{c|cccccc|c} 
0: FUL & 144 & $\infty$ & $\infty$ & $\infty$ & $\infty$ & $\infty 4.2 e 4$ & $0 / 15$ \\
1: AVG & 241 & $\infty$ & $\infty$ & $\infty$ & $\infty$ & $\infty 3.1 \mathrm{e} 4$ & $0 / 15$ \\
\hline f111 & 6856 & $6.12 \mathrm{e} 5$ & $8.83 \mathrm{e} 6$ & $2.30 \mathrm{e} 7$ & $3.10 \mathrm{e} 7$ & $3.13 \mathrm{e} 7$ & $3 / 15$ \\
0: FUL & $\infty$ & $\infty$ & $\infty$ & $\infty$ & $\infty$ & $\infty 4.2 \mathrm{e} 4$ & $0 / 15$
\end{tabular}

\begin{tabular}{c|cccccc|c}
1 : AVG & $\infty$ & $\infty$ & $\infty$ & $\infty$ & $\infty$ & $\infty 4.2 e 4$ & $0 / 15$ \\
\hline$f_{112}$ & 107 & 1684 & 3421 & 4502 & 5132 & $\infty 3.1 e 4$ & $0 / 15$ \\
\hline FU & 1 & & 18 & & & $\infty 56$ & $15 / 15$
\end{tabular}

\begin{tabular}{c|cccccc|c}
$0:$ FUL & 1 & 6.3 & 18 & $\infty$ & $\infty$ & $\infty 4.3 e 4$ & $0 / 15$ \\
$1:$ AVG & 3.1 & 4.9 & 23 & $\infty$ & $\infty$ & $\infty 3.3 e 4$ & $0 / 15$ \\
\hline$f_{113}$ & 133 & 1883 & 8081 & 24128 & 24128 & 24402 & $15 / 15$
\end{tabular}

\begin{tabular}{c|cccccc|c}
\hline f $_{113}$ & 133 & 1883 & 8081 & 24128 & 24128 & 24402 & $15 / 15$ \\
O: FUL & 19 & 57 & $\infty$ & $\infty$ & $\infty$ & $\infty 4.2 e 4$ & $0 / 15$ \\
\hline : AVV & 14 & 31 & 55 & $\infty$ & $\infty$ &
\end{tabular}

\begin{tabular}{c|cccccc|c} 
1: AVG & 14 & 31 & 55 & $\infty$ & $\infty$ & $\infty 3.1 e 4$ & $0 / 15$ \\
\hline f $114_{114}$ & 767 & 14720 & 56311 & 83272 & 83272 & 84949 & $15 / 15$
\end{tabular}

\begin{tabular}{l|cccccc|c} 
0: FUL & 60 & $\infty$ & $\infty$ & $\infty$ & $\infty$ & $\infty 4.2 e 4$ & $0 / 15$ \\
1: AVG & 74 & $\infty$ & $\infty$ & $\infty$ & $\infty$ & $\infty 3.1 e 4$ & $0 / 15$ \\
\hline
\end{tabular}

\begin{tabular}{l|lccccc|c}
\hline f115 & 64 & 485 & 1829 & 2550 & 2550 & 2970 & $15 / 15$ \\
0: FUL & 1 & 2.8 & 17 & $\infty$ & $\infty$ & $\infty 4.0 e 4$ & $0 / 15$ \\
1. AVG & 1.1 & 4.2 & 28 & $\infty$ & $\infty$ & $\infty 3.0 e 4$ & $0 / 15$ \\
\hline
\end{tabular}

\begin{tabular}{l|rrrrrr|r} 
1: AVG & 1.1 & 4.2 & 28 & $\infty$ & $\infty$ & $\infty 3.0 e 4$ & $0 / 15$ \\
\hline$f_{116}$ & 5730 & 14472 & 22311 & 26868 & 30329 & 31661 & $15 / 15$
\end{tabular}

\begin{tabular}{c|cccccc|c} 
0: FUL & $\infty$ & $\infty$ & $\infty$ & $\infty$ & $\infty$ & $\infty 4.2 \mathrm{e} 4$ & $0 / 15$ \\
$1:$ AVG & $\infty$ & $\infty$ & $\infty$ & $\infty$ & $\infty$ & $\infty 3.1 \mathrm{e} 4$ & $0 / 15$ \\
\hline $\mathrm{f}_{117}$ & 26686 & 76052 & $1.10 \mathrm{e} 5$ & $1.37 \mathrm{e} 5$ & $1.73 \mathrm{e} 5$ & $1.92 \mathrm{e} 5$ & $15 / 15$
\end{tabular}

\begin{tabular}{c|cccccc|c}
\hline f117 & 26686 & 76052 & $1.10 \mathrm{e} 5$ & $1.37 \mathrm{e} 5$ & $1.73 \mathrm{e} 5$ & $1.92 \mathrm{e} 5$ & $15 / 15$ \\
0: FUL & $\infty$ & $\infty$ & $\infty$ & $\infty$ & $\infty$ & $\infty 4.2 e 4$ & $0 / 15$ \\
1: AVG & $\infty$ & $\infty$ & $\infty$ & $\infty$ & $\infty$ & $\infty 3.1 e 4$ & $0 / 15$ \\
\hline
\end{tabular}

\begin{tabular}{c|cccccc|c}
1 : AVG & $\infty$ & $\infty$ & $\infty$ & $\infty$ & $\infty$ & $\infty 3.1 e 4$ & $0 / 15$ \\
\hline f118 & 429 & 1217 & 1555 & 1998 & 2430 & 2913 & $15 / 15$ \\
0: FUL & 1.3 & 5.9 & 70 & $\infty$ & $\infty$ & $\infty 4.3 e 4$ & $0 / 15$
\end{tabular}

\begin{tabular}{c|cccccc|c}
0 : FULG & 1.8 & 8.3 & 64 & $\infty$ & $\infty$ & $\infty 3.4 e 4$ & $0 / 15$ \\
\hline : AVG & 12 & 657 & 1136 & 10372 & 35296 & 49747 & $15 / 15$
\end{tabular}

\begin{tabular}{c|ccccccc|c} 
0: FUV & 19 & 23 & $\infty$ & $\infty$ & $\infty$ & $\infty 4.2 e 4$ & $0 / 15$ \\
\hline : AV & 16 & 2900 & 18698 & 72438 & $3.33 \mathrm{e} 5$ & $5.48 \mathrm{e} 4$ & $0 / 15$ \\
\hline f120 $_{12}$ & 16 & $15 / 15$
\end{tabular}

\begin{tabular}{l|cccccc|c} 
0: FUL & 151 & 63 & $\infty$ & $\infty$ & $\infty$ & $\infty 4.3 e 4$ & $0 / 15$ \\
1: AVG & 94 & 49 & $\infty$ & $\infty$ & $\infty$ & $\infty 3.1 e 4$ & $0 / 15$ \\
\hline$f_{121}$ & 8.6 & 111 & 273 & 1583 & 3870 & 6195 & $15 / 15$ \\
0. FUL & 3.2 & 10 & 33 & $\infty$ & $\infty$ & $\infty 4.0 e 4$ & $0 / 15$
\end{tabular}

\begin{tabular}{ccccccc|c} 
0: FUL & 3.2 & 10 & 33 & $\infty$ & $\infty$ & $\infty 4.0 e 4$ & $0 / 15$ \\
1: AVG & 4.3 & 3.3 & 45 & $\infty$ & $\infty$ & $\infty 3.0 e 4$ & $0 / 15$ \\
\hline f 122 & 10 & 1727 & 9190 & 30087 & 53743 & $1.11 \mathrm{e} 5$ & $15 / 15$ \\
0: FUL & 30 & 110 & $\infty$ & $\infty$ & $\infty$ & $\infty 4.2 e 4$ & $0 / 15$
\end{tabular}

\begin{tabular}{c|cccccc|c}
$1:$ AVG & 6.1 & 34 & $\infty$ & $\infty$ & $\infty$ & $\infty 3.1 e 4$ & $0 / 15$ \\
\hline$f_{123}$ & 11 & 16066 & 81505 & $3.36 \mathrm{e} 5$ & $6.71 \mathrm{e} 5$ & $2.22 \mathrm{e} 6$ & $15 / 15$
\end{tabular}

\begin{tabular}{l|cccccc|c} 
0: FUL & 113 & 18 & $\infty$ & $\infty$ & $\infty$ & $\infty 4.3 e 4$ & $0 / 15$ \\
1.: AVG & 80 & $\infty$ & $\infty$ & $\infty$ & $\infty$ & $\infty .124$ & $0 / 15$ \\
\hline
\end{tabular}

\begin{tabular}{c|cccccc|c}
1. AVG & 80 & $\infty$ & $\infty$ & $\infty$ & $\infty$ & $\infty 3.1 e 4$ & $0 / 15$ \\
\hline f $124_{12}$ & 10 & 202 & 1040 & 20478 & 45337 & 95200 & $15 / 15$ \\
0: FUL & 5.3 & 45 & $\infty$ & $\infty$ & $\infty$ & $\infty 3.9 e 4$ & $0 / 15$
\end{tabular}

\begin{tabular}{c|cccccc|c}
1 : AVG & 6.1 & 89 & $\infty$ & $\infty$ & $\infty$ & $\infty 2.9 e 4$ & $0 / 15$ \\
\hline$f_{125}$ & 1 & 1 & 1 & $2.39 \mathrm{e} 5$ & $2.43 \mathrm{e} 5$ & $2.46 \mathrm{e} 5$ & $15 / 15$
\end{tabular}

\begin{tabular}{l|cccccc|c} 
0: FUL & 3.9 & 26 & 5566 & $\infty$ & $\infty$ & $\infty 4.2 e 4$ & $0 / 15$ \\
1: AVG & 2.0 & 81 & 9966 & $\infty$ & $\infty$ & $\infty 3.1 e 4$ & $0 / 15$ \\
\hline
\end{tabular}

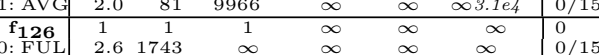

\begin{tabular}{c|cccccc|c} 
1: AVG & 22 & 1636 & $4.54 \mathrm{e} 5$ & $\infty$ & $\infty$ & $\infty$ & $0 / 15$ \\
\hline$f_{127}$ & 1 & 1 & 1 & $3.42 \mathrm{e} 5$ & $3.89 \mathrm{e} 5$ & $3.95 \mathrm{e} 5$ & $15 / 15$
\end{tabular}

\begin{tabular}{l|llrlll|l} 
0: FUL & 4.1 & 25 & 10666 & $\infty$ & $\infty$ & $\infty 3.9 e 4$ & $0 / 15$ \\
1: AVG & 2.0 & $\mathbf{1 8}^{\star}$ & 5233 & $\infty$ & $\infty$ & $\infty 2.9 e 4$ & $0 / 15$ \\
\hline
\end{tabular}

\begin{tabular}{c|cccccc|c}
1. AVG & 2.0 & $\mathbf{1 8}^{\star}$ & 5233 & $\infty$ & $\infty$ & $\infty 2.9 e 4$ & $0 / 15$ \\
\hline f $128_{12}$ & 111 & 4248 & 7808 & 12447 & 17217 & 21162 & $15 / 15$ \\
0. FUL & 15 & 11 & 36 & $\infty$ & $\infty$ & $\infty 42 e 4$ & $0 / 15$
\end{tabular}

\begin{tabular}{l|rrrrrr|r} 
1: AVG & 11 & 9.3 & 7.5 & $\infty$ & $\infty$ & $\infty 3.2 \mathrm{e}$ & $0 / 15$ \\
\hline$f_{129}$ & 64 & 10710 & 59443 & $2.85 \mathrm{e} 5$ & $5.11 \mathrm{e} 5$ & $5.80 \mathrm{ee} 5$ & $15 / 15$ \\
\hline
\end{tabular}

\begin{tabular}{ccccccc|c} 
P $_{129}$ & 64 & 10710 & 59443 & 2 & $\infty$ & $\infty$ & $\infty$ \\
$0:$ FUL & 128 & 58 & $\infty$ & $\infty$ & $\infty$ & $\infty 4.3 e 4$ & $0 / 1$ \\
\hline
\end{tabular}

\begin{tabular}{l|cccccc|c}
$1:$ AVG & 68 & 20 & $\infty$ & $\infty$ & $\infty$ & $\infty 3.1 e 4$ & $0 / 15$ \\
\hline$f_{130}$ & 55 & 812 & 3034 & 32823 & 33889 & 34528 & $10 / 15$
\end{tabular}

\begin{tabular}{l|llllll|l} 
1: AVG & 3.0 & 7.0 & 3.0 & 2.4 & $\infty$ & $\infty 4.0 e 4$ & $0 / 15$ \\
& 1.3 & 6.4 & 5.9 & 3.0 & $\infty$ & $\infty 3.0 e 4$ & $0 / 15$
\end{tabular}
20-D

\begin{tabular}{|c|c|c|c|c|c|c|c|}
\hline$\Delta f$ & $1 \mathrm{e}+1$ & $1 e+0$ & $1 \mathrm{e}-1$ & $1 \mathrm{e}-3$ & $1 e-5$ & $1 \mathrm{e}-7$ & \# suco \\
\hline & 59 & 361 & 513 & 700 & 739 & 783 & $15 / 15$ \\
\hline & 5.8 & 1.7 & 1.3 & 1 & 1 & 1 & $15 / 15$ \\
\hline & $3.3^{\star 3}$ & $1.2^{\star 3}$ & $0.95^{\star 3}$ & 0.94 & 1.0 & 1.1 & $15 / 15$ \\
\hline & 231 & 399 & 579 & 921 & 1157 & 1407 & $15 / 15$ \\
\hline & 1.6 & 1.7 & 1.3 & 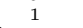 & 1 & 1 & $15 / 15$ \\
\hline AVG & $0.92^{\star 3}$ & $1.1^{\star 3}$ & $0.93^{\star 2}$ & 0.88 & 1.1 & 1.3 & $15 / 15$ \\
\hline & 65 & 417 & 629 & 1313 & 1893 & 2464 & $14 / 15$ \\
\hline & 5.2 & 1.5 & 1.1 & $7.8^{\star 2}$ & $97^{\star 3}$ & $554^{\star 3}$ & $1 / 15$ \\
\hline & $3.0^{\star 3}$ & $0.95^{\star 3}$ & 2.0 & 655 & $\infty$ & $\infty 2.0 e 5$ & $0 / 15$ \\
\hline & 23690 & 85656 & $1.71 \mathrm{e} 5$ & $1.82 \mathrm{e}$ & $1.89 \mathrm{c}$ & $5 \quad 1.96 \mathrm{e} 5$ & $15 / 15$ \\
\hline
\end{tabular}

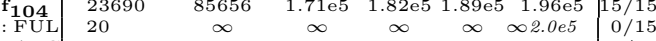

\begin{tabular}{c|cccccc|c} 
1: AVG & 11 & $\infty$ & $\infty$ & $\infty$ & $\infty$ & $\infty 2.0 e 5$ & $0 / 15$ \\
\hline f $_{105}$ & $1.92 \mathrm{e} 5$ & $6.11 \mathrm{e} 5$ & $6.32 \mathrm{e} 5$ & $6.49 \mathrm{e} 5$ & $6.60 \mathrm{e} 5$ & $6.70 \mathrm{e} 5$ & $15 / 15$
\end{tabular}

\begin{tabular}{l|cccccc|c} 
0: FUL & 7.2 & $\infty$ & $\infty$ & $\infty$ & $\infty$ & $\infty 2.0 e 5$ & $0 / 15$ \\
1: AVG & 7.2 & $\infty$ & $\infty$ & $\infty$ & $\infty$ & $\infty 2.0 e 5$ & $0 / 15$ \\
\hline f 106 & 11480 & 21668 & 23746 & 25470 & 26492 & 27360 & $15 / 15$ \\
0. FUL & 3.3 & 12 & 28 & & & $\infty$ & $\infty$
\end{tabular}

\begin{tabular}{ccccccc|c} 
0: FUL & 3.3 & 12 & 28 & $\infty$ & $\infty$ & $\infty 2.0 e 5$ & $0 / 15$ \\
1: AVG & 8.1 & $\infty$ & $\infty$ & $\infty$ & $\infty$ & $\infty 2.0 e 5$ & $0 / 15$ \\
\hline f 107 & 8571 & 13582 & 16226 & 27357 & 52486 & 65052 & $15 / 15$ \\
0: FUL & $\infty$ & $\infty$ & $\infty$ & $\infty$ & $\infty$ & $\infty 2.0 e 5$ & $0 / 15$
\end{tabular}

\begin{tabular}{l|cccccc|c} 
0: FUL & $\infty$ & $\infty$ & $\infty$ & $\infty$ & $\infty$ & $\infty 2.0 \mathrm{e} 5$ & $0 / 15$ \\
1: AVG & $\infty$ & $\infty$ & $\infty$ & $\infty$ & $\infty$ & $\infty 1.8 \mathrm{e} 5$ & $0 / 15$ \\
\hline $\mathbf{f}_{108}$ & 58063 & 97228 & $2.03 \mathrm{e} 5$ & $4.46 \mathrm{e} 5$ & $6.30 \mathrm{e} 5$ & $8.98 \mathrm{e} 5$ & $15 / 15$
\end{tabular}

\begin{tabular}{l|cccccc|c} 
0: FUL & $\infty$ & $\infty$ & $\infty$ & $\infty$ & $\infty$ & $\infty 2.0 e 5$ & $0 / 15$ \\
1: AVG & $\infty$ & $\infty$ & $\infty$ & $\infty$ & $\infty$ & $\infty 1.8 \mathrm{e} 5$ & $0 / 15$ \\
\hline$f_{109}$ & 333 & 632 & 1138 & 2287 & 3583 & 4952 & $15 / 15$
\end{tabular}

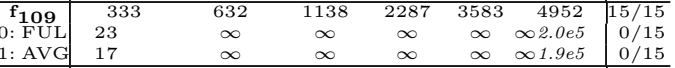

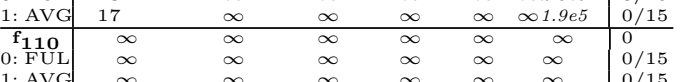

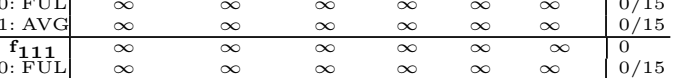

\begin{tabular}{ccccccc|c} 
: FUL & $\infty$ & $\infty$ & $\infty$ & $\infty$ & $\infty$ & $\infty$ & $0 / 15$ \\
1: AVG & $\infty$ & $\infty$ & $\infty$ & $\infty$ & $\infty$ & $\infty$ & $0 / 15$ \\
\hline f $112_{112}$ & 25552 & 64124 & 69621 & 73557 & 76137 & 78238 & $15 / 15$
\end{tabular}

\begin{tabular}{l|cccccc|c}
\hline f 112 & 25552 & 64124 & 69621 & 73557 & 76137 & 78238 & $15 / 15$ \\
0: FUL & $\infty$ & $\infty$ & $\infty$ & $\infty$ & $\infty$ & $\infty 2.0 e 5$ & $0 / 15$ \\
1: AVG & $\infty$ & $\infty$ & $\infty$ & $\infty$ & $\infty$ & $\infty 2.0 e 5$ & $0 / 15$ \\
\hline
\end{tabular}

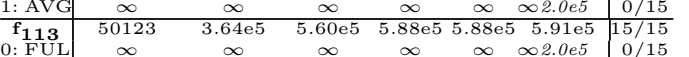

\begin{tabular}{ccccccc|c} 
0: FUL & $\infty$ & $\infty$ & $\infty$ & $\infty$ & $\infty$ & $\infty 2.0 \mathrm{ee} 5$ & $0 / 15$ \\
1: AVG & $\infty$ & $\infty$ & $\infty$ & $\infty$ & $\infty$ & $\infty 1.8 \mathrm{e} 5$ & $0 / 15$ \\
\hline $\mathbf{f}_{114}$ & $2.08 \mathrm{e} 5$ & $1.12 \mathrm{e} 6$ & $1.45 \mathrm{e} 6$ & $1.57 \mathrm{e} 6$ & $1.57 \mathrm{e} 6$ & $1.58 \mathrm{e} 6$ & $15 / 15$
\end{tabular}

\begin{tabular}{l|cccccc|c}
\hline f 114 & $2.08 \mathrm{e} 5$ & $1.12 \mathrm{e} 6$ & $1.45 \mathrm{e} 6$ & $1.57 \mathrm{e} 6$ & $1.57 \mathrm{e} 6$ & $1.58 \mathrm{e} 6$ & $15 / 15$ \\
0: FUL & $\infty$ & $\infty$ & $\infty$ & $\infty$ & $\infty$ & $\infty 2.0 \mathrm{e} 5$ & $0 / 15$ \\
1: AVG & $\infty$ & $\infty$ & $\infty$ & $\infty$ & $\infty$ & $\infty 1.8 \mathrm{e} 5$ & $0 / 15$ \\
\hline
\end{tabular}

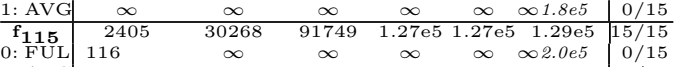

\begin{tabular}{c|cccccc|c} 
0: FUL & 116 & $\infty$ & $\infty$ & $\infty$ & $\infty$ & $\infty 2.0 e 5$ & $0 / 15$ \\
1: AVG & 108 & $\infty$ & $\infty$ & $\infty$ & $\infty$ & $\infty 1.9 \mathrm{e} 5$ & $0 / 15$ \\
\hline f116 & $4.98 \mathrm{e} 5$ & $6.94 \mathrm{e} 5$ & $8.93 \mathrm{e} 5$ & $1.03 \mathrm{e} 6$ & $1.08 \mathrm{e} 6$ & $1.12 \mathrm{e} 6$ & $15 / 15$ \\
0: FUL & $\infty$ & $\infty$ & $\infty$ & $\infty$ & $\infty$ & $\infty 2.0 \mathrm{e} 5$ & $0 / 15$
\end{tabular}

\begin{tabular}{ccccccc|c}
$1:$ AVG & $\infty$ & $\infty$ & $\infty$ & $\infty$ & $\infty$ & $\infty 1.8 \mathrm{e} 5$ & $0 / 15$ \\
\hline f 117 & $1.79 \mathrm{e} 6$ & $2.46 \mathrm{e} 6$ & $2.60 \mathrm{e} 6$ & $2.91 \mathrm{e} 6$ & $3.24 \mathrm{e} 6$ & $3.62 \mathrm{e} 6$ & $15 / 15$ \\
$0:$ FUL & $\infty$ & $\infty$ & $\infty$ & $\infty$ & $\infty$ & $\infty 2.0 \mathrm{e} 5$ & $0 / 15$
\end{tabular}

\begin{tabular}{c|cccccc|c} 
0: FUL & $\infty$ & $\infty$ & $\infty$ & $\infty$ & $\infty$ & $\infty 2.0 e 5$ & $0 / 15$ \\
1: AVG & $\infty$ & $\infty$ & $\infty$ & $\infty$ & $\infty$ & $\infty 1.8 e 5$ & $0 / 15$ \\
\hline 118 & 6908 & 11786 & 17514 & 26342 & 30062 & 32659 & $15 / 15$ \\
1 FUL & $\infty$ & $\infty$ & $\infty$ & $\infty$ & $\infty$ & $\infty 2.0 e 5$ & $0 / 15$
\end{tabular}

\begin{tabular}{l|cccccc|c}
\hline 118 & 6908 & 11786 & 17514 & 26342 & 30062 & 32659 & $15 / 15$ \\
0: FUL & $\infty$ & $\infty$ & $\infty$ & $\infty$ & $\infty$ & $\infty 2.0 \mathrm{e} 5$ & $0 / 15$ \\
1: AVG & $\infty$ & $\infty$ & $\infty$ & $\infty$ & $\infty$ & $\infty 2.0 \mathrm{e} 5$ & $0 / 15$ \\
\hline
\end{tabular}

\begin{tabular}{c|cccccc|c}
\hline f119 & 2771 & 29365 & 35930 & $4.11 \mathrm{e} 5$ & $1.40 \mathrm{e} 6$ & $1.90 \mathrm{e} 6$ & $15 / 15$ \\
0: FUL & 1046 & $\infty$ & $\infty$ & $\infty$ & $\infty$ & $\infty 2.0 \mathrm{e} 5$ & $0 / 15$
\end{tabular}

\begin{tabular}{c|cccccc|c} 
0: FUL & 1046 & $\infty$ & $\infty$ & $\infty$ & $\infty$ & $\infty 2.0 \mathrm{e} 5$ & $0 / 15$ \\
1: AVG & $\infty$ & $\infty$ & $\infty$ & $\infty$ & $\infty$ & $\infty 1.8 \mathrm{e} 5$ & $0 / 15$ \\
\hline f 120 & 36040 & $1.79 \mathrm{e} 5$ & $2.81 \mathrm{e} 5$ & $1.59 \mathrm{e} 6$ & $6.74 \mathrm{e} 6$ & $1.35 \mathrm{e} 7$ & $13 / 15$ \\
0: FUL & $\infty$ & $\infty$ & $\infty$ & $\infty$ & $\infty$ & $\infty 2.0 \mathrm{e} 5$ & $0 / 15$ \\
1: AVG & $\infty$ & $\infty$ & $\infty$ & & $\infty$ & $\infty 1.8 \mathrm{e} 5$ & $0 / 15$
\end{tabular}

\begin{tabular}{c|cccccc|c} 
1: AVG & $\infty$ & $\infty$ & $\infty$ & $\infty$ & $\infty$ & $\infty 1.8 e 5$ & $0 / 15$ \\
\hline f $121_{121}$ & 249 & 769 & 1426 & 9304 & 34434 & 57404 & $15 / 15$ \\
: FUL & 73 & $\infty$ & $\infty$ & $\infty$ & $\infty$ & $\infty 2.0 e 5$ & $0 / 15$
\end{tabular}

\begin{tabular}{c|cccccc|c}
$1: \mathrm{AVG}$ & 49 & $\infty$ & $\infty$ & $\infty$ & $\infty$ & $\infty 2.0 \mathrm{e} 5$ & $0 / 15$ \\
\hline $\mathbf{f}_{122}$ & 692 & 52008 & $1.40 \mathrm{e} 5$ & $7.93 \mathrm{e} 5$ & $2.00 \mathrm{e} 6$ & $5.82 \mathrm{e} 6$ & $15 / 15$ \\
\hline
\end{tabular}

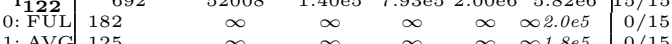

\begin{tabular}{c|cccccc|l} 
1: AVG & 125 & $\infty$ & $\infty$ & $\infty$ & $\infty$ & $\infty 1.8 \mathrm{e} 5$ & $0 / 15$ \\
\hline $\mathbf{f}_{123}$ & 1063 & $5.30 \mathrm{e} 5$ & $1.49 \mathrm{e} 6$ & $5.29 \mathrm{e} 6$ & $2.71 \mathrm{e} 7$ & $1.58 \mathrm{e} 8$ & 0
\end{tabular}

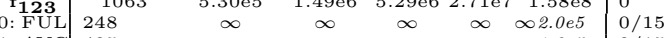

\begin{tabular}{c|cccccc|c} 
1: AVG & 405 & $\infty$ & $\infty$ & $\infty$ & $\infty$ & $\infty 1.8 \mathrm{e} 5$ & $0 / 15$ \\
\hline $\mathbf{f}_{124}$ & 192 & 1959 & 40840 & $1.27 \mathrm{e} 5$ & $3.89 \mathrm{e} 5$ & $7.99 \mathrm{e} 5$ & $15 / 15$
\end{tabular}

\begin{tabular}{l|cccccc|c} 
0: FUL & 117 & $\infty$ & $\infty$ & $\infty$ & $\infty$ & $\infty 2.0 e 5$ & $0 / 15$ \\
1: AVG & 95 & $\infty$ & $\infty$ & $\infty$ & $\infty$ & $\infty 1.8$ e 5 & $0 / 15$ \\
\hline
\end{tabular}

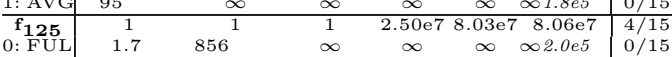

\begin{tabular}{l|cccccc|c} 
1: AVG & 1 & 856 & $\infty$ & $\infty$ & $\infty$ & $\infty 2.0 e 5$ & $0 / 15$ \\
\hline$f_{126}$ & 1 & 1 & 1 & $\infty$ & $\infty$ & $\infty$ & 0
\end{tabular}

\begin{tabular}{l|l}
\hline 126 & 11 \\
\hline : FUL & 311 \\
\hline
\end{tabular}

\begin{tabular}{llcllll|l}
$1:$ AVG & 122 & $2.60 \mathrm{e} 6$ & $\infty$ & $\infty$ & $\infty$ & $\infty$ & $0 / 15$ \\
\hline$f$ & 1 & & $\infty$ & $\infty$ & $\infty$ & $0 / 15$ \\
\hline
\end{tabular}

\begin{tabular}{c|cccccc|c} 
f127 & 1 & 1 & 1 & $4.43 \mathrm{e} 6$ & $7.27 \mathrm{e} 6$ & $7.43 \mathrm{e} 6$ & $15 / 15$ \\
$0:$ FUL & 1 & 1360 & $\infty$ & $\infty$ & $\infty$ & $\infty 2.0 \mathrm{e} 5$ & $0 / 15$
\end{tabular}

\begin{tabular}{c|cccccc|c}
$1: \mathrm{AVG}$ & 7.7 & $\mathbf{2 1 9 ^ { \star 2 }}$ & $\infty$ & $\infty$ & $\infty$ & $\infty 2.0 \mathrm{e} 5$ & $0 / 15$ \\
\hline $\mathbf{f}_{128}$ & $1.40 \mathrm{e} 5$ & $1.34 \mathrm{e} 7$ & $1.72 \mathrm{e} 7$ & $1.72 \mathrm{e} 7$ & $1.72 \mathrm{e} 7$ & $1.72 \mathrm{e} 7$ & $0 / 15$ \\
\hline
\end{tabular}

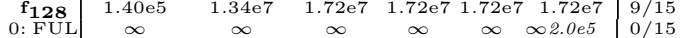

\begin{tabular}{c|cccccc|c}
$1: \mathrm{AVG}$ & $\infty$ & $\infty$ & $\infty$ & $\infty$ & $\infty$ & $\infty 1.8 \mathrm{e} 5$ & $0 / 15$ \\
\hline $\mathrm{f}_{129}$ & $7.81 \mathrm{e} 6$ & $4.13 \mathrm{e} 7$ & $4.15 \mathrm{e} 7$ & $4.18 \mathrm{e} 7$ & $4.21 \mathrm{e} 7$ & $4.24 \mathrm{e} 7$ & $5 / 15$
\end{tabular}

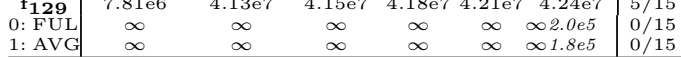

\begin{tabular}{c|cccccc|c} 
1: AVG & $\infty$ & $\infty$ & $\infty$ & $\infty$ & $\infty$ & $\infty 1.8 \mathrm{e} 5$ & $0 / 15$ \\
\hline $\mathbf{f}_{130}$ & 4904 & 93149 & $2.52 \mathrm{e} 5$ & $2.54 \mathrm{e} 5$ & $2.55 \mathrm{e} 5$ & $2.57 \mathrm{e} 5$ & $7 / 15$
\end{tabular}

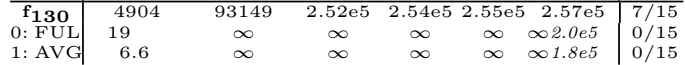

Table 2: ERT in number of function evaluations divided by the best ERT measured during BBOB-2009 (given in the respective first row) for different $\Delta f$ values for functions $f_{101}-f_{130}$. \#succ is the number of trials that reached the final target $f_{\mathrm{opt}}+10^{-8}$. 0: FUL is full-NEWUOA and 1: AVG is avg-NEWUOA. \#succ is the number of trials that reached the final target $f_{\mathrm{opt}}+10^{-8}$. 0: FUL is full-NEWUOA and 1: AVG is avgNEWUOA. Bold entries are statistically significantly better compared to the other algorithm, with $p=0.05$ or $p=10^{-k}$ where $k>1$ is the number following the $\star$ symbol, with Bonferroni correction of 60 . 